\title{
Broadcast copies reveal the quantumness of correlations
}

\author{
M. Piani, ${ }^{1}$ M. Christandl, ${ }^{2}$ C. E. Mora, ${ }^{1}$ and P. Horodecki ${ }^{3,4}$ \\ ${ }^{1}$ Institute for Quantum Computing $\&$ Department of Physics and Astronomy, \\ University of Waterloo, 200 University Ave. W., N2L 3G1 Waterloo, Canada \\ ${ }^{2}$ Faculty of Physics, Ludwig-Maximilians-Universität München, Theresienstrasse 37, 80333 Munich, Germany \\ ${ }^{3}$ Faculty of Applied Physics and Mathematics, Gdańsk University of Technology, 80-952 Gdańsk, Poland \\ ${ }^{4}$ National Quantum Information Centre of Gdańsk, 81-824 Sopot, Poland
}

\begin{abstract}
We study the quantumness of bipartite correlations by proposing a quantity that combines a measure of total correlations - mutual information- with the notion of broadcast copies -i.e. generally non-factorized copies- of bipartite states. By analyzing how our quantity increases with the number of broadcast copies, we are able to classify classical, separable, and entangled states. This motivates the definition of the broadcast regularization of mutual information, the asymptotic minimal mutual information per broadcast copy, which we show to have many properties of an entanglement measure.
\end{abstract}

PACS numbers:

Much work has recently been performed in order to analyze how correlations can be understood, quantified and classified as either classical or quantum [1, 2]. Such studies, that in a way go beyond the standard entangledversus-separable [3] distinction, are relevant not only for our understanding of the fundamental differences between the classical and quantum world, but also from the point of view of Quantum Information Processing [4]. Indeed, Entanglement is a necessary prerequisite for a task such as Quantum Key Distribution [5], but its role in Quantum Computation is less clear, as there are cases where quantum correlations that are weaker than entanglement seem to be sufficient to boost performance with respect to classical computation [6].

In studying the quantumness of correlations, researchers have focused on the following hierarchy of states. Classical-classical (CC-) states are of the form $\sum_{i j} p_{i j}^{A B}|i\rangle\left\langle\left. i\right|_{A} \otimes \mid j\right\rangle\left\langle\left. j\right|_{B}\right.$, with $\left\{|i\rangle_{A}\right\}$ and $\left\{|j\rangle_{B}\right\}$ orthonormal bases, and $\left\{p_{i j}^{A B}\right\}$ a joint probability distribution. A CC-state is the embedding of a probability distribution in the formalism of quantum theory and as such has no quantumness. CC-states are a proper subclass the class of separable states, which are of the form $\sum_{k} p_{k} \rho_{A}^{k} \otimes \rho_{B}^{k}$ for a probability distribution $\left\{p_{k}\right\}$ and local quantum states $\rho_{A}^{k}$ and $\rho_{B}^{k}$. Separable states can be generated with Local Operations and Classical Communication (LOCC) only and are therefore considered to have little quantumness. The remaining states are called entangled and exhibit the most quantumness.

In this Letter we study the quantumness of correlations by combining a measure of total correlations mutual information (MI) - and the notion of broadcast copies, i.e. generally non-factorized copies (see Figure 1) of a bipartite quantum state. We relate quantumness to monogamy of correlations, and in particular to monogamy of entanglement, which in standard terms refers to the impossibility of a system to be strongly entangled with two or more other systems at the same time [7]. Here, we adopt a different perspective by con- sidering broadcast copies, and analyze quantitatively the minimal growth of the correlations with the number of broadcast copies. Whereas for factorized copies the correlations increase linearly for all states, this is not true for non-factorized copies. Indeed, for CC-states correlations do not have to increase at all and can be freely shared among any number of broadcast copies. We show that for non-CC separable states there is actually an increase, but it is bounded, while for entangled states the correlations must increase linearly with the copies, a result we term copy-copy monogamy of entanglement. This is better expressed in quantitative terms by introducing the broadcast regularization of MI, the minimal per-copy MI between parties, when they share an infinite amount of broadcast copies. We show that this quantity has many properties of an entanglement measure [8], we establish relations with known entanglement measure, and we conjecture that it is an entanglement measure itself. We then restrict the minimization to permutationallyinvariant broadcast copies and prove that the corresponding constrained broadcast regularization of MI equals the classical version of squashed entanglement [9].

Broadcast copies and mutual information. In [2] the quantumness of correlations of a bipartite state $\rho_{A B}$ on Hilbert space $\mathcal{H}_{A} \otimes \mathcal{H}_{B}$ was addressed from an operational point of view by employing the notion of broadcast copies and by quantifying total correlations by means of MI. The MI of a state $\rho \equiv \rho_{A B}$ is defined as $I(\rho) \equiv I(A: B)_{\rho} \equiv S(A)_{\rho}+S(B)_{\rho}-S(A B)_{\rho}$, with $S(X)_{\rho}=-\operatorname{Tr} \rho_{X} \log _{2} \rho_{X}$ the von Neumann entropy of subsystem $X$ when the state of the total system is $\rho$ [21]. We say that a state $\rho_{X^{n}}^{(n)}, X^{n} \equiv X_{1} \ldots X_{n}$, is an $n$-copy broadcast state of $\rho$ if $\rho_{X_{k}}^{(n)} \equiv \operatorname{Tr}_{X_{1} \cdots X_{k-1} X_{k+1} \cdots X_{n}} \rho^{(n)}=\rho$ for all $k$. Each system $X_{k}$ may be composed of subsystems, in our case $X_{k}=A_{k} B_{k}$. Broadcast copies may contain correlations among the different copies, in contrast to factorized copies $\rho_{X}^{\otimes n}$ (Figure 1). For example, given any mixed ensemble $\left\{\left(p_{k}, \rho_{A B}^{k}\right)\right\}$ for $\rho_{A B}$, 


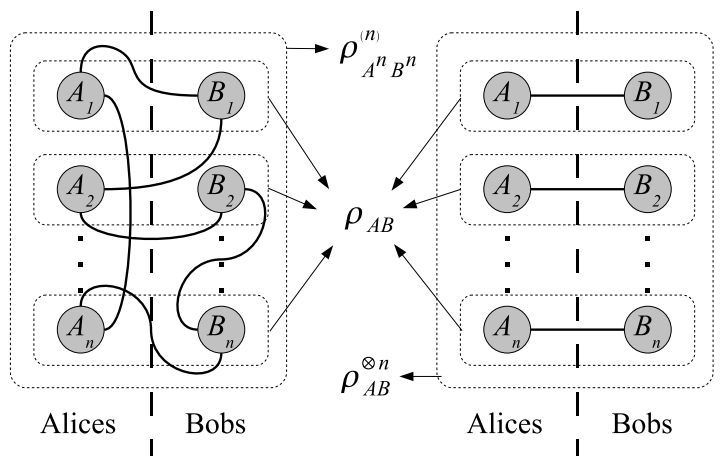

FIG. 1: $n$ copies of a bipartite state $\rho_{A B}$ : broadcast copies (left) and factorized copies (right). Solid lines connecting subsystems symbolize correlations. The vertical dashed line indicates the bipartite cut across which correlations are quantified by mutual information.

i.e. $\rho_{A B}=\sum_{k} p_{k} \rho_{A B}^{k}$, the convex combination of factorized states $\rho^{(n)}\left[\left\{\left(p_{k}, \rho_{A B}^{k}\right)\right\}\right] \equiv \sum_{k} p_{k} \rho_{A B}^{k}{ }^{\otimes n}$ is a possible $n$-copy broadcast state of $\rho_{A B}$. Such states are also known as de Finetti states and play an important role in quantum versions of de Finetti's theorem [10, 11]. By combining MI and the notion of broadcast copies, we can define the $n$-copy broadcast $M I$ of $\rho_{A B}$ as

$$
\left(I_{b}\right)_{n}\left(\rho_{A B}\right) \equiv \min _{\rho^{(n)}} I\left(A^{n}: B^{n}\right)_{\rho^{(n)}},
$$

where the minimum is taken over all $n$-copy broadcast states $\rho_{A^{n} B^{n}}^{(n)}$ of $\rho_{A B}$. A broadcast copy $\bar{\rho}^{(n)}$ such that $I\left(\bar{\rho}^{(n)}\right)=\left(I_{b}\right)_{n}\left(\rho_{A B}\right)$ will be said to be optimal [22]. In [2] a no-local-broadcasting theorem for quantum correlations was derived by proving that for non-CC stateseven separable ones - one has $\left(I_{b}\right)_{n}\left(\rho_{A B}\right)>I\left(\rho_{A B}\right)$, for $n \geq 2$. This suggests that the quantumness of the correlations present in $\rho_{A B}$ may be revealed by the dependence of $\left(I_{b}\right)_{n}\left(\rho_{A B}\right)$ on the number of broadcast copies $n$ [23]. We will particularly focus on its behaviour for large $n$, as given by the broadcast regularization of MI [24]

$$
I_{b}^{(\infty)}\left(\rho_{A B}\right) \equiv \lim _{n} \frac{1}{n} \min _{\rho^{(n)}} I\left(A^{n}: B^{n}\right)_{\rho^{(n)}} .
$$

In the following theorem we formalize the intuition that classical correlations can be freely shared among the broadcast copies, while quantum correlations can not.

Theorem 1. The $n$-copy broadcast $M I\left(I_{b}\right)_{n}$ as a function of $n$ : (i) is constant for CC-states; (ii) grows (strictly from one to two copies) but is bounded for separable states that are not CC-states; (iii) grows strictly and asymptotically linearly for all entangled states.

Proof. (ii) By definition, given any mixed ensemble realization $\left\{\left(p_{k}, \rho_{A B}^{k}\right)\right\}$ of $\rho_{A B}$, we have $\left(I_{b}\right)_{n}\left(\rho_{A B}\right) \leq$ $I\left(\rho^{(n)}\left[\left\{\left(p_{k}, \rho_{A B}^{k}\right)\right\}\right]\right)$. For separable states one may choose an ensemble with $\rho_{A B}^{k}=\rho_{A}^{k} \otimes \rho_{B}^{k}$, for all $k$. Then, independently of the number of copies $n$, $I\left(\rho^{(n)}\left[\left\{\left(p_{k}, \rho_{A}^{k} \otimes \rho_{B}^{k}\right)\right\}\right]\right) \leq S\left(\left\{p_{k}\right\}\right) . \quad$ In the separable non-CC case, the strict growth from $n=1$ to $n=2$ of $\left(I_{b}\right)_{n}$ was proved in [2]. (i) For CC-states, one can relabel $k=(i, j)$ and set $\rho_{A B}^{k} \equiv\left|i_{A}\right\rangle\left\langle i_{A}|\otimes| j_{B}\right\rangle\left\langle j_{B}\right|$, so that $I\left(\rho_{A B}\right)=\left(I_{b}\right)_{n}\left(\rho_{A B}\right)=I\left(\rho^{(n)}\left[\left\{\left(p_{i j},\left|i_{A}\right\rangle\left\langle i_{A}\right| \otimes\right.\right.\right.\right.$ $\left.\left.\left.\left.\left|j_{B}\right\rangle\left\langle j_{B}\right|\right)\right\}\right]\right)=I\left(\left\{p_{i j}\right\}\right)[25]$ which is a constant independent of $n$. (iii) By definition, $I_{b}^{(\infty)}=\lim _{n} \frac{1}{n}\left(I_{b}\right)_{n}$, therefore $\left(I_{b}\right)_{n} \geq n I_{b}^{(\infty)}[26$. The claim follows then from the statement that $I_{b}^{(\infty)}$ is strictly positive for all entangled states (Lemma 10 below).

Properties of $I_{b}^{(\infty)}$. The next theorem establishes many of the properties of $I_{b}^{(\infty)}$.

Theorem 2. The broadcast regularization of $M I I_{b}^{(\infty)}$ is: (i) zero for separable states; (ii) convex; (iii) monotone under local operations: $I\left(\left(\Lambda_{A} \otimes \Lambda_{B}\right)\left[\rho_{A B}\right]\right) \leq I\left(\rho_{A B}\right)$, for completely-positive trace-preserving maps $\Lambda_{A}$ and $\Lambda_{B}$; (iv) subadditive: $I_{b}^{(\infty)}\left(\rho_{A B} \otimes \sigma_{A^{\prime} B^{\prime}}\right) \leq I_{b}^{(\infty)}\left(\rho_{A B}\right)+$ $I_{b}^{(\infty)}\left(\sigma_{A^{\prime} B^{\prime}}\right) ; \quad(v)$ weakly additive: $I_{b}^{(\infty)}\left(\rho_{A B}^{\otimes m}\right)=$ $m I_{b}^{(\infty)}\left(\rho_{A B}\right)$; (vi) asymptotically continuous: for $\epsilon \equiv$ $\left\|\rho_{A B}-\sigma_{A B}\right\|_{1}<\left(\frac{2}{21}\right)^{2},\|X\|_{1}=\operatorname{Tr} \sqrt{X^{\dagger} X}$, one has $\left|I_{b}^{(\infty)}\left(\rho_{A B}\right)-I_{b}^{(\infty)}\left(\sigma_{A^{\prime} B^{\prime}}\right)\right| \leq 126 \sqrt{\epsilon} \log _{2} d+6 h\left(\frac{21}{2} \sqrt{\epsilon}\right)$, with $h(x)=-x \log _{2} x-(1-x) \log _{2}(1-x)$ and $d$ the dimension of $A B$.

Proof. (i) is a consequence of Theorem 1. (ii) is proved by noting that for optimal broadcast copies $\bar{\rho}_{i}^{(n)}$ of $\rho_{i}: \quad\left(I_{b}\right)_{n}\left(\sum_{i} p_{i} \rho_{i}\right) \leq I\left(\sum_{i} p_{i} \rho_{i}^{(n)}\right) \leq \sum_{i} p_{i}\left(I_{b}\right)_{n}\left(\rho_{i}\right)+$ $S\left(\left\{p_{i}\right\}\right)$. (iii) derives from the fact that if $\bar{\rho}^{(n)}$ is an optimal broadcast copy for $\rho$, then $\left(\Lambda_{A}^{\otimes n} \otimes \Lambda_{B}^{\otimes n}\right)\left[\bar{\rho}^{(n)}\right]$ is a broadcast copy of $\left(\Lambda_{A} \otimes \Lambda_{B}\right)\left[\rho_{A B}\right]$ and $\left(I_{b}\right)_{n}\left(\left(\Lambda_{A} \otimes\right.\right.$ $\left.\left.\Lambda_{B}\right)\left[\rho_{A B}\right]\right) \leq I\left(\left(\Lambda_{A}^{\otimes n} \otimes \Lambda_{B}^{\otimes n}\right)\left[\bar{\rho}^{(n)}\right]\right) \leq I\left(\bar{\rho}^{(n)}\right)=\left(I_{b}\right)_{n}(\rho)$. (iv) follows from the additivity of MI: $\left(I_{b}\right)_{n}(\rho \otimes \sigma) \leq$ $I\left(\bar{\rho}^{(n)} \otimes \bar{\sigma}^{(n)}\right)=\left(I_{b}\right)_{n}(\rho)+\left(I_{b}\right)_{n}(\sigma)$, for $\bar{\rho}^{(n)}$ and $\bar{\sigma}^{(n)}$ optimal broadcast copies of $\rho$ and $\sigma$, respectively. Given subadditivity, in order to prove (v) it is sufficient to observe that $I_{b}^{(\infty)}\left(\rho^{\otimes k}\right)=\lim _{m} \frac{1}{m}\left(I_{b}\right)_{m}\left(\rho^{\otimes k}\right) \geq$ $k \lim _{m} \frac{1}{m k}\left(I_{b}\right)_{m k}(\rho)=k I_{b}^{(\infty)}(\rho)$. The proof of (vi) is relatively technical and will be reported elsewhere [12]. The main idea is to first prove that for any $\rho_{X}, \sigma_{X}$, $\epsilon \equiv\left\|\rho_{X}-\sigma_{X}\right\|_{1}<1$, there exist a quantum operation $\Lambda_{X} \equiv \Lambda_{X}(\rho, \sigma)$ such that: (a) $\Lambda_{X}\left[\rho_{X}\right]=\sigma_{X}$, and (b) for any extension $\tau_{X Y}$ satisfying $\tau_{X}=\rho_{X}$, $\left\|\left(\Lambda_{X} \otimes \mathbb{1}_{Y}\right)\left[\tau_{X Y}\right]-\tau_{X Y}\right\| \leq \frac{21}{2} \sqrt{\epsilon}$. Thus, if $\rho^{(n)}$ is a broadcast copy of $\rho$, there exist $\Lambda$ such that $\sigma^{(n)}=\Lambda^{\otimes n}\left[\rho^{(n)}\right]$ is a broadcast copy of $\sigma$ with comparable MI.

According to Theorem 2, $I_{b}^{(\infty)}$ has many of the properties of an entanglement measure [8], and we conjecture that it really is an entanglement monotone, i.e. that it decreases (on average) under LOCC. 
Relation to entanglement measures. A way to prove that $I_{b}^{(\infty)}>0$ for all entangled states is suggested by noting the relation of $I_{b}^{(\infty)}$ to known entanglement measures $[9,13,14,15]$ : squashed entanglement $E_{\mathrm{sq}}^{Q}\left(\rho_{A B}\right) \equiv$ $\left.\frac{1}{2} \inf _{\rho_{A B E}}\left(I(A: B E)_{\rho}-I(A: E)\right)_{\rho}\right)$, where the infimum is over all extensions $\rho_{A B E}$ of $\rho_{A B}$, i.e. states $\rho_{A B E}$ satisfying $\operatorname{Tr}_{E}\left(\rho_{A B E}\right)=\rho_{A B}$; conditional entanglement of $M I$ (CEMI) $E_{I}\left(\rho_{A B}\right) \equiv \frac{1}{2} \inf _{\rho_{A B A^{\prime} B^{\prime}}}\left(I\left(A A^{\prime}: B B^{\prime}\right)_{\rho}-I\left(A^{\prime}\right.\right.$ : $\left.\left.\left.B^{\prime}\right)\right)_{\rho}\right)$, with the infimum over extensions of $\rho_{A B}$; classical squashed entanglement $E_{\mathrm{sq}}^{C}\left(\rho_{A B}\right) \equiv \frac{1}{2} \min _{\rho_{A B \hat{E}}}(I(A$ : $\left.\left.B \hat{E})_{\rho}-I(A: \hat{E})\right)_{\rho}\right)$, where the minimum is over all extensions $\rho_{A B \hat{E}}$ of $\rho_{A B}$ that are classical on $\hat{E}$, i.e. $\rho_{A B \hat{E}}=\sum_{k} p_{k} \rho_{A B}^{k} \otimes|k\rangle\left\langle\left. k\right|_{\hat{E}}\right.$. Squashed entanglement and CEMI obey $E_{I} \geq E_{\mathrm{sq}}^{Q}$, and have an operational interpretation as minimal quantum communication costs in quantum state redistribution [14, 15].

By the definition of $E_{I}\left(\rho_{A B}\right)$, it holds $I\left(A A^{\prime}: B B^{\prime}\right)_{\rho} \geq$ $2 E_{I}\left(\rho_{A B}\right)+I\left(A^{\prime}: B^{\prime}\right)_{\rho}$ for any extension $\rho_{A A^{\prime} B B^{\prime}}$ of $\rho_{A B}$. Therefore, given an $n$-copy broadcast state $\rho_{A B}^{(n)}$, by using recursively the broadcast condition one obtains $\left(I_{b}\right)_{n}\left(\rho_{A B}\right) \geq 2 n E_{I}\left(\rho_{A B}\right)$. By dividing both sides of this inequality by $n$ and taking the limit $n \rightarrow \infty$, we get $I_{b}^{(\infty)} \geq 2 E_{I}$. Nonetheless, neither $E_{I}$ nor $E_{\mathrm{sq}}^{Q}$ are known to be strictly positive for all entangled states, in particular because the extending systems in the definitions may have any dimension. Interestingly, thanks to the classicality of the extension, $E_{\mathrm{sq}}^{C}$ has a finite-dimensional optimal extension $\hat{E}$ and is thus known to be strictly positive for all entangled states [9].

In order to find good lower bounds on $I_{b}^{(\infty)}$ we consider the classical MI associated to a bipartite state quantum $\rho_{A B}$ [16], defined as $I_{C}\left(\rho_{A B}\right) \equiv$ $\max _{\left\{M_{i} \otimes N_{j}\right\}} I\left(\left\{p_{i j}\left(\rho_{A B}\right)\right\}\right)$. The maximum is taken with respect to all local POVMs $M_{i} \geq 0, \sum_{i} M_{i}=\mathbb{1}$ (acting on system $A$ ) and $N_{j} \geq 0, \sum_{i} N_{j}=\mathbb{1}$, (acting on system $B$ ) respectively, and $p_{i j}(\rho)=\operatorname{Tr}\left(M_{i} \otimes N_{j} \rho\right)$. As MI decreases under local measurements, $I_{C}\left(\rho_{A B}\right) \leq I\left(\rho_{A B}\right)$, with equality if and only if the state $\rho_{A B}$ is CC [2]. We now define a quantity in the same spirit of $E_{I}$ :

$$
\left.E_{I_{C}}\left(\rho_{A B}\right) \equiv \inf _{\rho}\left(I_{C}\left(A A^{\prime}: B B^{\prime}\right)_{\rho}-I_{C}\left(A^{\prime}: B^{\prime}\right)\right)_{\rho}\right),
$$

with $\rho \equiv \rho_{A B A^{\prime} B^{\prime}}$ an extension of $\rho_{A B} . E_{I_{C}}$ measures the minimal increase in classical correlations due to "adding" two systems $A B$ in the state $\rho_{A B}$ to arbitrary ancillas $A^{\prime} B^{\prime}$. The following lemma proves that $E_{I_{C}}\left(\rho_{A B}\right)>0$ if and only if $\rho_{A B}$ is entangled: entanglement and only entanglement implies a higher amount of classical correlations. Furthermore, it relates $I_{b}^{(\infty)}$ and $E_{I_{C}}$ and completes the proof of Theorem 1 .

Lemma 1. It holds that (i) $I_{b}^{(\infty)} \geq E_{I_{C}}$, and that (ii) $E_{I_{C}}$ vanishes for and only for separable states.

Proof. (i) For any $n$-copy broadcast state $\rho^{(n)}$ of $\rho$, we have $I\left(\rho^{(n)}\right) \geq I_{C}\left(\rho^{(n)}\right) \geq n E_{I_{C}}(\rho)$, where we used again the broadcast condition and the definition of $E_{I_{C}}$. Thus, $I_{b}^{(\infty)} \geq\left(I_{C}\right)_{b}^{(\infty)} \geq E_{I_{C}}$. (ii) The latter relations prove that $E_{I_{C}}$ vanishes for separable states. In order to prove strict positivity on entangled states, consider any extension $\rho_{A B A^{\prime} B^{\prime}}$ of a state $\rho_{A B}$. The optimal local measurements for $I_{C}\left(\rho_{A B A^{\prime} B^{\prime}}\right)$ in general act jointly on $A A^{\prime}$ and $B B^{\prime}$. Let us restrict ourselves to measurements $\left\{M_{k}^{\prime}\right\}$ and $\left\{N_{l}^{\prime}\right\}$ on $A^{\prime}$ and $B^{\prime}$ that attain the maximum in $I_{C}\left(\rho_{A^{\prime} B^{\prime}}\right)$, and optimize solely over POVMs $\left\{M_{i}\right\}$ and $\left\{N_{j}\right\}$ on $A$ and $B$. Thus, as in the first part of the proof of Theorem 3 in [2], by using the definition of $\mathrm{MI}$ and the concavity of entropy we find $I_{C}\left(\rho_{A B A^{\prime} B^{\prime}}\right)-$ $I_{C}\left(\rho_{A^{\prime} B^{\prime}}\right) \geq \sup _{\left\{M_{i} \otimes N_{j}\right\}} \sum_{k l} q_{k l} I\left(\left\{p_{i j}\left(\rho_{A B}^{k l}\right)\right\}\right)$, with $q_{k l} \equiv \operatorname{Tr}\left(\rho_{A^{\prime} B^{\prime}} M_{k}^{\prime} \otimes N_{l}^{\prime}\right)$ and $\rho_{A B}^{k l}=\operatorname{Tr}_{A^{\prime} B^{\prime}}\left(\rho_{A B A^{\prime} B^{\prime}} M_{k}^{\prime} \otimes\right.$ $\left.N_{l}^{\prime}\right) / q_{k l}\left(\rho_{A^{\prime} B^{\prime}}\right)$. We now recall that MI can be expressed as relative entropy [28]. Furthermore, $p_{i}^{A}\left(\rho_{A B}^{k l}\right) \equiv$ $\sum_{j} p_{i j}\left(\rho_{A B}^{k l}\right)=\operatorname{Tr}_{A}\left(M_{i} \rho_{A}^{k l}\right)$, with $\rho_{A}^{k l}=\operatorname{Tr}_{B}\left(\rho_{A B}^{k l}\right)$ (similarly for $\left.p_{j}^{B}\left(\rho_{A B}^{k l}\right)\right)$. Thus, we find

$$
\begin{aligned}
& \sup _{\left\{M_{i} \otimes N_{j}\right\}} \sum_{k l} q_{k l} I\left(\left\{p_{i j}\left(\rho_{A B}^{k l}\right)\right\}\right) \\
& \geq \sup _{\left\{M_{i} \otimes N_{j}\right\}} S\left(\left\{p_{i j}\left(\rho_{A B}\right)\right\} \|\left\{p_{i j}\left(\sum_{k l} q_{k l} \rho_{A}^{k l} \otimes \rho_{B}^{k l}\right)\right\}\right) \\
& \geq \inf _{\sigma_{A B} \text { separable } \sup _{\left\{M_{i} \otimes N_{j}\right\}} S\left(\left\{p_{i j}\left(\rho_{A B}\right)\right\} \|\left\{p_{i j}\left(\sigma_{A B}\right)\right\}\right),}
\end{aligned}
$$

where we used the joint convexity of relative entropy, the fact that $\sum_{k l} q_{k l} \rho_{A B}^{k l}=\rho_{A B}$, as well as the separability of $\sum_{k l} q_{k l} \rho_{A}^{k l} \otimes \rho_{B}^{k l}$. This lower bound is independent of $\rho_{A B A^{\prime} B^{\prime}}$ and is strictly positive for all entangled states, because there exist informationally-complete local POVMs [19] and the relative entropy vanishes only when the two probability distributions are equal.

The next theorem formalizes the relation of $I_{b}^{(\infty)}$ with the mentioned entanglement measures.

Theorem 3. We have the sequence of inequalities:

$$
2 E_{\mathrm{sq}}^{C} \geq 2\left(E_{\mathrm{sq}}^{C}\right)^{(\infty)} \geq I_{b}^{(\infty)} \geq 2 E_{I} \geq 2 E_{\mathrm{sq}}^{Q} .
$$

Proof. The two rightmost inequalities have already been discussed. The first inequality is due to subadditivity of $E_{\mathrm{sq}}^{C}$. The inequality $2 E_{\mathrm{sq}}^{C} \geq I_{b}^{(\infty)}$ is proved by noticing that $E_{\mathrm{sq}}^{C}$ corresponds to $E_{\mathrm{sq}}^{C}\left(\rho_{A B}\right)=$ $\frac{1}{2} \min _{\left\{\left(p_{k}, \rho_{A B}^{k}\right)\right\}} \sum_{k} p_{k} I\left(\rho_{A B}^{k}\right)$, with the minimum over mixed ensembles for $\rho_{A B}$ [9]. By choosing an ensemble $\left\{\left(\bar{p}_{k}, \bar{\rho}_{A B}^{k}\right)\right\}$ optimal for $E_{\text {sq }}^{C}$, and using additivity of MI, one finds $\left(I_{b}\right)_{n}\left(\rho_{A B}\right) \leq I\left(\rho^{(n)}\left[\left\{\left(\bar{p}_{k}, \bar{\rho}_{A B}^{k}\right)\right\}\right]\right) \leq$ $n \sum_{k} \bar{p}_{k} I\left(\bar{\rho}_{A B}^{k}\right)+S\left(\left\{\bar{p}_{k}\right\}\right)=2 n E_{\mathrm{sq}}^{C}\left(\rho_{A B}\right)+S\left(\left\{\bar{p}_{k}\right\}\right)$. The second inequality in (11) is obtained by the standard regularization of both sides of $2 E_{\mathrm{sq}}^{C} \geq I_{b}^{(\infty)}$.

Theorem 3 together with Lemma 1, provides a new proof that the entanglement $\operatorname{cost} E_{c}$ - the rate at which one has to consume pure entanglement to create many 
copies of a given state via LOCC - is strictly positive for all entangled states [17]. Indeed, by using the formula for $E_{c}$ of [18], one easily checks that $E_{c} \geq\left(E_{\mathrm{sq}}^{C}\right)^{(\infty)}$.

Finally, we notice that one may define a variant of $I_{b}^{(\infty)}$ by considering broadcast copies only in the class of permutation-invariant states, that is, states $\rho_{A^{n} B^{n}}^{(n)}$ satisfying $\rho_{A^{n} B^{n}}^{(n)}=\pi \rho_{A^{n} B^{n}}^{(n)} \pi^{-1}$, for all permutations $\pi$ of the $n$ pairs $A_{i} B_{i}$. Thus, we define the symmetrical broadcast regularization of $M I$ as:

$$
I_{b, \text { sym }}^{(\infty)}\left(\rho_{A B}\right) \equiv \lim _{n} \frac{1}{n} \operatorname{\rho in}_{\rho^{(n)}} \underset{\text { perm-inv }}{ } I\left(A^{n}: B^{n}\right)_{\rho^{(n)}} .
$$

For such a quantity we are able to establish the following.

Theorem 4. $I_{b, \mathrm{sym}}^{(\infty)}=2 E_{\mathrm{sq}}^{C}$, i.e., symmetric copies of the form $\rho^{(n)}\left[\left\{\left(p_{k}, \rho_{A B}^{k}\right)\right\}\right]$ are asymptotically optimal.

This theorem can be interpreted as support for our conjecture, since it implies that the symmetric version of $I_{b}^{(\infty)}$ is an entanglement monotone.

By Theorem 3 it suffices to prove the direction " $\geq$ ". The intuition is that permutation-invariant states can be approximated by de Finetti states [10, 11]. This idea can be made precise with the help of the so-called exponential de Finetti theorem, in particular by showing that entropy is "robust" under the disturbance of a small number of subsystems [10] (see [12] for details).

Lemma 2. Let $\rho_{A^{n} B^{n}}$ be a permutation-invariant state on $\left(\mathcal{H}_{A} \otimes \mathcal{H}_{B}\right)^{\otimes n}$. Then there exists an ensemble of states $\left\{p_{i}, \rho_{A B}^{i}\right\}$, where $\rho_{A B}^{i}$ are states on $\mathcal{H}_{A} \otimes \mathcal{H}_{B}$, such that $S\left(X^{n}\right)_{\rho_{A^{n} B^{n}}}=n \sum_{i} p_{i} S(X)_{\rho_{A B}^{i}}+o(n)$, for $X=A, B, A B$, and $\left\|\rho_{A B}-\sum_{i} p_{i} \rho_{A B}^{i}\right\|_{1}=o(1)$, with the reduced state $\rho_{A B}=\rho_{A_{k} B_{k}}, k=1, \ldots, n$.

Theorem 4 follows because for any permutationinvariant broadcast copy $\rho^{(n)}$ of $\rho$, the continuity of $E_{\mathrm{sq}}^{C}$ [9] and Lemma 2 assure the existence of an ensemble $\left\{\left(p_{i}, \sigma_{i}^{A B}\right)\right\}$ such that $I\left(\rho^{(n)}\left[\left\{\left(p_{i}, \sigma_{i}^{A B}\right)\right\}\right]\right) \leq I\left(\rho^{(n)}\right)+$ $o(n)$.

Conclusions. In this Letter, we have introduced a new way of quantifying the quantumness of correlations. This led us to define a new correlation measure, the broadcast regularization of mutual information. Its strict positivity on and only on entangled states can be interpreted as a signature of the monogamy of entanglement for any entangled state. Our study furthermore reveals a novel relation between extensions - here broadcast extensions and entanglement, a topic of practical interest [20].

Focus has been on correlations between two parties. As in [2], our results can be straightforwardly extended to the multipartite case if a suitable definition of multipartite mutual information is adopted.

We thank M. Horodecki, R. Renner, and B. Toner for discussions. This work began when MP was a Lise Meitner Fellow at the University of Innsbruck and is partially supported by the Austrian Science Fund (FWF).
We acknowledge support: by QuantumWorks and Ontario Centres of Excellence [MP and CM]; by the Excellence Network of Bavaria (TMP, QCCC) and the SFB 1388 of the German Science Foundation [MC]; by EU IP SCALA and the LFPPI network $[\mathrm{PH}]$.

[1] H. Ollivier and W.H. Zurek, Phys. Rev. Lett. 88, 017901 (2001); L. Henderson and V. Vedral, J. Phys. A: Math. Gen. 34, 6899 (2001); J. Oppenheim et al., Phys. Rev. Lett. 89, 180402 (2002); M. Horodecki et al., Phys. Rev. A 71, 062307 (2005); B. Groisman et al., Phys. Rev. A 72, 032317 (2005); B. Groisman et al., arXiv:quant-ph/0703103 N. Li and S. Luo, Phys. Rev. A 76, 032327 (2007).

[2] M. Piani et al., Phys. Rev. Lett. 100, 090502 (2008).

[3] R. Horodecki et al., arXiv:quant-ph/0702225 [Rev. Mod. Phys. (to be published)].

[4] M. A. Nielsen and I. L. Chuang, "Quantum Computation and Quantum Information", Cambridge University Press, Cambridge (2000).

[5] M. Curty et al., Phys. Rev. Lett. 92, 217903 (2004).

[6] E. Knill and R. Laflamme, Phys. Rev. Lett. 81, 5672 (1998); A. Datta et al., Phys. Rev. A 72, 042316 (2005); A. Datta et al., Phys. Rev. Lett. 100, 050502 (2008).

[7] V. Coffman et al., Phys. Rev. A 61, 052306 (2000).

[8] M. B. Plenio and S. Virmani, Quant. Inf. Comp. 7, 1 (2007)

[9] D. Yang et al., arXiv:0704.2236.

[10] R. Renner, Nature Physics 3, 645 (2007); R. Renner, PhD Thesis (2005), quant-ph/0512258. R. König and G. Mitchison, quant-ph/0703210 (2007).

[11] E. Størmer, J. Funct. Anal. 3, 48 (1969); R. L. Hudson and G. R. Moody, Z. Wahrschein. verw. Geb. 33, 343 (1976); C. M. Caves et al., J. Math. Phys. 43, 4537 (2002); R. König and R. Renner, J. Math. Phys. 46, 122108 (2005); M. Christandl et al., Comm. Math. Phys. 273, 473 (2007)

[12] M. Piani, M. Christandl, C. E. Mora, and P. Horodecki, in preparation.

[13] M. Christandl and A. Winter, J. Math. Phys. 45, 829 (2004).

[14] J. Oppenheim, arXiv:0801.0458

[15] D. Yang et al., Phys. Rev. Lett. 101, 140501 (2008).

[16] B. Terhal et al., J. Math. Phys. 43, 4286 (2002).

[17] D. Yang et al., Phys. Rev. Lett. 95, 190501 (2005).

[18] P. M. Hayden et al., J. Phys. A 34, 6891 (2001).

[19] C. M. Caves et al., J. Math. Phys. 43, 4537 (2002).

[20] G. O. Myhr et al., arXiv:0812.3607, A. C. Doherty et al., Phys. Rev. A 69, 022308 (2004).

[21] MI is a fundamental measure of correlations (see [1] and references therein) that: does not increase under local operations; is neither convex nor concave, but respects $I\left(\sum_{k} p_{k} \rho_{A B}^{k}\right) \leq \sum_{k} p_{k} I\left(\rho_{A B}^{k}\right)+S\left(\left\{p_{k}\right\}\right)$, with $S\left(\left\{p_{k}\right\}\right) \equiv-\sum_{k} p_{k} \log _{2} p_{k}$ the Shannon entropy of the classical probability distribution $\left\{p_{k}\right\}$; is additive: $I\left(\rho_{A_{1} B_{1}} \otimes \sigma_{A_{2} B_{2}}\right)=I\left(\rho_{A_{1} B_{1}}\right)+I\left(\sigma_{A_{2} B_{2}}\right)$.

[22] As the set of $n$-copy broadcast states is compact, there exist optimal broadcast copies.

[23] Notice that $I\left(\rho_{A B}\right) \leq\left(I_{b}\right)_{n}\left(\rho_{A B}\right) \leq I\left(\rho_{A B}^{\otimes n}\right)=n I\left(\rho_{A B}\right)$.

[24] The broadcast regularization of any a state-dependent 
real function $f$ will be denoted by $f_{b}^{(\infty)}$. The standard regularization $f^{(\infty)}$ is defined as $f^{(\infty)}\left(\rho_{A B}\right) \equiv$ $\lim _{n} \frac{1}{n} f\left(\rho_{A B}^{\otimes n}\right)$, i.e. on factorized copies. For MI, one has trivially $I^{(\infty)}=I$.

[25] Here, $I\left(\left\{p_{i j}^{A B}\right\}\right) \equiv S\left(\left\{p_{i}^{A}\right\}\right)+S\left(\left\{p_{j}^{B}\right\}\right)-S\left(\left\{p_{i j}^{A B}\right\}\right) \equiv$ $S\left(\left\{p_{i j}^{A B}\right\} \|\left\{p_{i}^{A} p_{j}^{B}\right\}\right)$ is the classical MI of the joint probability distribution $\left\{p_{i j}^{A B}\right\}$ with marginal distributions
$p_{i}^{A}=\sum_{j} p_{i j}^{A B} \quad($ similarly for $B) . \quad S\left(\left\{p_{k}\right\} \|\left\{q_{k}\right\}\right) \equiv$ $-\sum_{k} p_{k} \log _{2} \frac{q_{k}}{p_{k}}$ is the Kullback-Leibler distance between two probability distributions $\left\{p_{k}\right\}$ and $\left\{q_{k}\right\}$.

[26] Take an optimal broadcast copy $\bar{\rho}^{(n)}$. Then $\left(I_{b}\right)_{n}(\rho)=$ $n \frac{I\left(\left(\bar{\rho}^{(n)}\right)^{\otimes k}\right)}{n k} \geq n \frac{\left(I_{b}\right)_{n k}(\rho)}{n k}$, and taking the limit $k \rightarrow \infty$ proves the claim. 